\title{
Small-Group Standardized Patient Encounter Improves Athletic Training Students' Psychosocial Intervention and Referral Skills
}

\author{
Stacy E. Walker, PhD, ATC*; Thomas G. Weidner, PhD, ATC*; Ashley B. Thrasher, EdD, ATC† \\ *School of Kinesiology, Ball State University, Muncie, IN; $\dagger$ Department of Health, Physical Education, \\ and Sport Sciences, Arkansas State University, Jonesboro
}

Context: Athletic trainers provide psychological support, counseling, intervention, and referral to patients during clinical practice. However, students are rarely exposed to real-life opportunities to develop these skills.

Objective: To determine if a small-group standardized patient (SP) encounter improved athletic training students' interpersonal communication, psychosocial intervention, and referral skills.

Design: Cohort.

Setting: One Midwestern university.

Patients or Other Participants: Thirty-nine (14 male, 25 female; age $=22 \pm 1.0$ years) senior athletic training students.

Intervention(s): The experimental group $(n=20)$ engaged in a small-group SP encounter to teach interpersonal communication, psychosocial intervention, and referral skills, in addition to normally scheduled classes and clinical education. The control group $(n=19)$ engaged only in routine classroom and clinical education. Both groups participated in an individual SP encounter to assess skills.

Main Outcome Measure(s): A 19-item dichotomous checklist (yes/no) assessed participants on their interpersonal communication, psychosocial intervention, and referral skills (eg, listened with interest, asked about eating habits and menstrual period) during the individual SP encounter. A Fisher exact test evaluated differences between the experimental and control group scores for each checklist item. Mann-Whitney $U$ tests were used to compare combined checklist scores between the experimental and control groups. A Bonferroni correction was performed to control for multiple comparisons.

Results: The experimental group experienced a significant increase in psychosocial intervention and referral skills $(U=$ $77.5, P=0.001)$, but there was no difference between the 2 groups on interpersonal communication skills $(U=138, P=$ .149). Participants in the experimental group asked the SP about coping strategies for stress more often than the control group (Fisher exact test $P<.001$ ).

Conclusions: A small-group SP encounter improved the participants' psychosocial intervention and referral skills but not their interpersonal communication skills. These results suggest a small-group SP encounter can provide learning experiences to better prepare athletic training students for clinical practice.

Key Words: Educational intervention, learning outcomes, engagement

Dr Walker is currently an Associate Professor of Athletic Training in the School of Kinesiology, Ball State University. Please address all correspondence to Stacy E. Walker, PhD, ATC, School of Kinesiology, Ball State University, Human Performance Building, Muncie, IN 47306. sewalker@bsu.edu.

\section{Full Citation:}

Walker SE, Weidner TG, Thrasher AB. Small-group standardized patient encounter improves athletic training students' psychosocial intervention and referral skills. Athl Train Educ J. 2016;11(1):38-44. 


\title{
Small-Group Standardized Patient Encounter Improves Athletic Training Students' Psychosocial Intervention and Referral Skills
}

\author{
Stacy E. Walker, PhD, ATC; Thomas G. Weidner, PhD, ATC; Ashley B. Thrasher, EdD, ATC
}

\section{INTRODUCTION}

Athletic trainers are often the first to identify, counsel, and refer patients with psychosocial issues such as disordered eating, depression, and/or substance abuse. ${ }^{1}$ Mental disorders (eg, depression, anxiety, substance abuse) in the United States occur frequently, with approximately half the population meeting the criteria for 1 or more disorders in their lifetime. One in 4 will meet the criteria in any given year. ${ }^{2}$ The occurrences of disordered eating, ${ }^{3}$ depression, ${ }^{4}$ anxiety, ${ }^{4}$ binge drinking, and substance abuse ${ }^{3,5,6}$ are high among college athletes. Because of the prevalence of psychological disorders, athletic trainers must be able to communicate effectively in order to confidently identify the signs and symptoms associated with various psychosocial issues and intervene and refer athletes to appropriate health care professionals. Psychosocial intervention involves sensitive mental and emotional issues with which shame, embarrassment, and even further emotional harm could be associated ${ }^{7}$; therefore, athletic trainers must be adequately prepared to recognize and provide appropriate care. However, many students receive little real-time practice or evaluation. ${ }^{8,9}$ One reason is that the staff athletic trainers typically interact with collegiate patients themselves. ${ }^{10}$ Athletic training students must develop and learn to integrate interpersonal communication skills into practical application, ${ }^{11}$ and decision making and skill integration should be evaluated in a manner very similar to real life. ${ }^{9}$ Because of the deeply personal nature of psychosocial intervention and referral, guided training, such as role play or a small-group standardized patient (SP) encounter, to develop the necessary interpersonal skills will benefit students and their future patients. ${ }^{12}$

Standardized patients have been used to provide medical students with opportunities to practice difficult communication and interpersonal skills for over 30 years. ${ }^{13}$ They also can provide consistent educational experiences and allow students to develop these skills to provide optimal patient care. An SP is an individual who has been trained to consistently (in a standardized fashion) portray a particular condition, injury, or illness for teaching and evaluation purposes. ${ }^{14}$ Use of simulations or SPs is recommended ${ }^{15}$ whenever students are unable to practice clinical proficiencies on real patients.

Instead of having students interact with SPs individually, we chose to examine a small-group SP encounter for teaching purposes. We feel athletic training educators could implement some small-group SP teaching encounters into their curricula and wanted to research their use in athletic training. Smallgroup SP encounters are less time consuming for faculty to develop and implement. As reported previously, ${ }^{16}$ implementing SPs requires personnel to develop the case, train the SPs, and evaluate each student individually, as well as facilities (eg, multiple examination settings equipped with video equipment) available for each student's experience. A small-group SP encounter is an effective teaching approach commonly used in medical schools, ${ }^{17,18}$ allowing students to interact with the SP in a variety of ways depending on the learning objectives. ${ }^{18}$ For this study we chose the time-in/time-out method ${ }^{19}$ in which students in a small group of 3 or 4 together evaluate an SP with a faculty member and preceptor present. If the students get confused or are unsure how to proceed, then a time-out can be called to allow for discussion and coaching with the faculty member and/or preceptor. When the students are ready to return to the patient then a time-in is called and the conversation with the SP can proceed from the point it left off or be "rewound" to an earlier stage of the encounter. This method provides students with immediate feedback from the faculty member, preceptor, peers, and SP.

This time-in/time-out method with an SP was previously found to increase athletic training students' confidence and reflection, as well as promoting peer learning during the encounter, but that study did not investigate the impact on behavior or skills. ${ }^{20}$ The purpose of this study was to determine if a small-group SP encounter improved athletic training students' interpersonal communication, psychosocial intervention, and referral skills.

\section{METHODS}

\section{Participants}

Senior athletic training students $(\mathrm{N}=39 ; 14$ male, 25 female; age $=22 \pm 1.0$ years) from one Midwestern university participated in this study. All participants had completed the psychology of injury course during fall semesters from 2006 to 2010 and were enrolled in a senior capstone course during the following spring semesters of 2008 to 2011. Before the psychology of injury course, none of the participants had any previous education in psychosocial intervention and referral. No students had completed their psychosocial intervention and referral clinical proficiency evaluation before this study.

\section{Procedures}

We used a quasi-experimental research design. Participants from the spring 2008 and 2010 semesters served as the experimental group, and participants from spring 2009 and 2011 served as the control group. We staggered the treatment every other year to minimize the threat to external validity caused by time. Participants in both the experimental (smallgroup SP encounter) and control groups were enrolled in normally scheduled classes for that semester and engaged in clinical education. Institutional review board approval was obtained before any data collection. Participants were recruited each semester by a graduate assistant during regularly scheduled class periods. For the experimental group $(\mathrm{n}=20)$, during the fifth week of the spring semester, participants engaged in a small-group SP encounter to learn psychosocial intervention and referral skills. Participants were randomly assigned to groups of 3 or 4 , and then interacted with an SP needing psychosocial intervention and referral. During and after the encounter, participants received verbal 
Figure. Semester timeline. Abbreviation: SP, standardized patient.

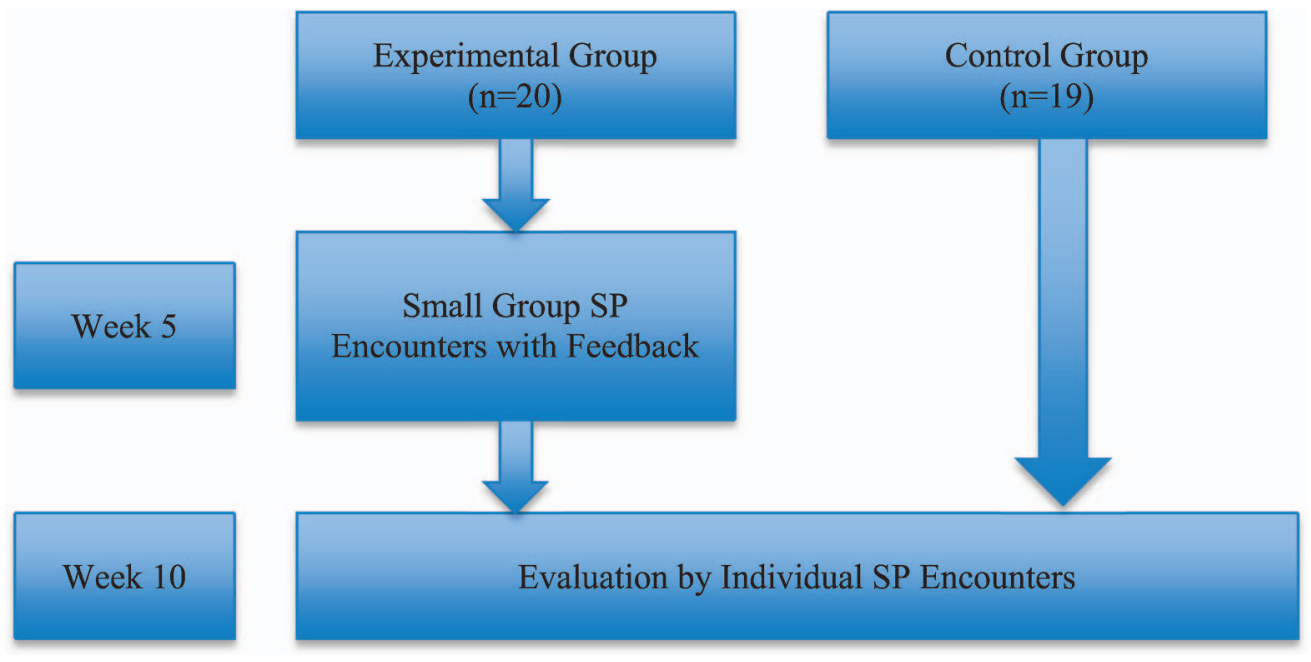

feedback from the SP, clinician/preceptor, and faculty member (S.E.W.), all of whom were present during all small-group SP encounters. The control group $(n=19)$ did not complete a small-group SP encounter. All participants in both the experimental and control groups engaged in an individual SP encounter approximately 10 weeks into the semester to determine if a small-group SP encounter improved athletic training students' interpersonal communication, psychosocial intervention, and referral skills. The Figure displays the semester timeline.

Small-Group SP Encounter. For the experimental group, a runny nose/sore throat cocaine abuse case was used during the small-group SP encounters. Participants were given basic information regarding the SP (eg, name, age, vitals, coach is concerned because of poor performance) and provided with a pen, a blank sheet of paper, and a clipboard. Participants were told this individual would be coming into the athletic training clinic and they together needed to evaluate and develop a plan for this patient. Their objective was to diagnose and treat the patient appropriately. The time-in/time-out method ${ }^{18}$ was explained to the participants.

The time-in/time-out method was used to provide immediate feedback during the SP encounter, on an as-needed basis. If students became too overwhelmed or uncertain on how to proceed, a time-out was called. Student participants, the faculty member, and/or the clinician/preceptor could call a time-out, but otherwise, the faculty member and clinician/ preceptor did not intervene or speak during the encounter. During this time-out, discussion took place among the SP (no longer in character), participants, faculty member, and/or clinician/preceptor. This discussion included dialogue about the participants' thought processes regarding patient care, possible next steps in the evaluation, appropriateness of questions and their phrasing, and a variety of other concerns or issues not normally discussed in the presence of a patient. When time-in was called, the participants then continued to interact with the SP (in character).

Once the participants felt they understood the procedures for the encounter, the encounter began. The participants, faculty member, and clinician/preceptor called time-outs for various reasons (eg, participants unsure how to proceed with asking about addiction or drug use or how to phrase certain questions, inaccurate history questions leading to inaccurate diagnosis). During time-out, participants asked questions and received feedback from the faculty member, clinician/preceptor, and SP. Once the small-group SP encounter ended, the participants, faculty member, clinician/preceptor, and SP together debriefed with each small group. During the debriefing, participants discussed their feelings, successes, and challenges during the encounter. Feedback from the faculty member, clinician/ preceptor, and SP focused primarily on interpersonal communication, psychosocial intervention, and referral skills. Only verbal feedback was provided to the students because this experience was to help students learn psychosocial intervention and referral skills and participants were not being formally evaluated. All small-group SP encounters lasted approximately 45 minutes to 1 hour and occurred in the Athletic Training Education and Research Laboratory.

Individual SP Encounters. Approximately 10 weeks (4 weeks after participants in the experimental group engaged in the small-group SP encounter) into the semester, all participants in both groups (experimental and control) were scheduled for an individual SP encounter (30 minutes). The case for this SP encounter was an individual with an eating disorder needing psychosocial intervention and referral. We chose 10 weeks because this gave the participants in the smallgroup SP encounter 4 weeks to reflect upon their experience and possibly to implement the skills learned during the smallgroup SP encounter in real time. Before participants interacted with the SP, the faculty member provided a short verbal summary of the procedures (eg, purpose of the SP encounter, how long encounter would last, what to do when encounter was over) and a clipboard with pen, paper, and relevant information (eg, name, age, complaint of muscle soreness). The faculty member answered any questions from the participant before each encounter.

All encounters took place in the Clinical Proficiency Evaluation Room, a mini-athletic training clinic with a treatment table and various medical supplies (eg, first aid, reflex hammer); the room was equipped with a camera and microphone in the ceiling. The faculty member watched on a TV monitor from an adjacent room and scored all encounters live using the Psychosocial Intervention and Referral Check- 
list. After the encounter, the SP scored all encounters using this same checklist. All participants were provided feedback on their performance from the faculty member and the SP immediately after the encounter. No clinician/preceptor was present during or after the encounter, as this SP encounter was to strictly evaluate participant performance.

Development of SP Cases. For the purposes of this study, $2 \mathrm{SP}$ cases were developed with the objective to provide a patient-centered psychosocial intervention and referral experience for the participants. The SP encounter for the experimental group involved a 21-year-old male with a complaint of a runny nose and sore throat (cocaine abuse). The individual SP encounter involved an 18-year-old female complaining of chronic muscular cramping during activity (eating disorder). Both cases were developed by the primary investigator (who has training and experience in development of cases), along with a clinician, using information from the literature and actual patient encounters in clinical practice. Both cases were reviewed and evaluated by an educator with SP research experience, an athletic training educator, and a clinician for difficulty level, accuracy of content, and/or authenticity.

Psychosocial Intervention and Referral Checklist. The Psychosocial Intervention and Referral Checklist (Table) was developed by the primary investigator and a clinician to evaluate all participants on their performance during the individual SP (eating disorder) encounter. Each checklist item was specific to the eating disorder SP case and identified appropriate interpersonal and psychosocial intervention and referral skills critical to that case for accurate diagnosis and referral. Participants' performance was scored with a yes or no on the checklist by both the SP and the faculty member. The checklist contained 2 sections: interpersonal communication (10 items) and psychosocial intervention and referral skills (9 items). The checklist, as previously described, was validated by an educator with SP research experience and an athletic training educator and clinician. Although we were not determining reliability of the checklist nor comparing the SP and faculty scores, interrater reliability was determined between the SP and the faculty member via intraclass correlation coefficient $([3,1]=0.641)$.

Training of SPs. Three theater students at the same institution were recruited to serve as SPs. All SPs underwent approximately 3 hours of training. The purpose was to train each of the SPs to portray the respective cases in a consistent fashion, and to provide feedback to the participants on their performance. For the cocaine abuse case, only $1 \mathrm{SP}$ was trained and used. For the eating disorder case, because of the number of participants and scheduling challenges, 2 SPs were trained and used. For all training, the SPs were provided with and reviewed the case information (same information from above for SP case development section) and the participant instructions. The 2 SPs trained on the eating disorder case also were provided with and reviewed the checklist. The SPs were then oriented as to the sequence of events (eg, time-in/time-out, feedback to be provided by the SP) for each patient encounter. After review of the case information, the SPs rehearsed the answers to questions and any required body language. SPs were instructed to provide positive and constructive feedback to the participants based on their own perspective as a patient (eg, I felt you were not listening, I felt you cared about my situation). SPs portraying the eating disorder case also provided feedback based on the items listed on the checklist.

\section{Data Analysis}

Descriptive statistics were calculated for all checklist items. The checklists scored by the faculty member were used in the data analysis. We were not interested in data obtained from the SP on the checklist items at this time, as their experience was rather limited. A Fisher exact test determined differences between the experimental and control group scores on the checklist for each checklist item. The Fisher exact test is used to assess differences between groups with categorical data. A Bonferroni correction was performed to control for multiple comparisons and reduce the risk of type I error, which set the $\alpha$ level at .003 for the Fisher exact test.

The checklist was then divided into 2 different subscales: interpersonal skills (questions 1-10) and psychosocial intervention and referral skills (questions 11-19). The scores were obtained by combining individual checklist items on each subscale. The dependent variables were the combined scores on each subscale (interpersonal skills and psychosocial intervention and referral skills). The independent variables were the 2 groups: experimental and control. Separate MannWhitney $U$ tests were performed to assess between-groups differences on each subscale. The Mann-Whitney $U$ test is appropriate for determining differences in nonparametric data. A Bonferroni correction was performed to control for multiple comparisons, which set the $\alpha$ level at .025 for the Mann-Whitney $U$ tests. The Statistical Package for the Social Sciences (version 21.0; SPSS Inc, Chicago, IL) was used for all statistical analyses.

\section{RESULTS}

The Fisher exact test $(\alpha=.003)$ revealed participants in the experimental group asked the SP about coping strategies for stress more often than participants in the control group (Fisher exact test $P=.001$ ). There were no significant differences between groups on the other individual checklist items. The checklist scores are presented in the Table. A Mann-Whitney $U$ test $(\alpha=.025)$ revealed a significant difference in the performance of the experimental group on the psychosocial intervention and referral skills $(U=77.5, P<$ .001 ), with those in the experimental group performing better than those in the control group. The experimental group correctly completed an average of $4.55 \pm 1.88$ out of 9 psychosocial and intervention referral skills, whereas the control group correctly completed on average $2.63 \pm 1.21$ items. No difference was found between experimental and control groups on the interpersonal communication skills subscale $(U=138, P=.149)$. The experimental group completed an average of $9.05 \pm 1.35$ out of 10 interpersonal communication skills, whereas the control group completed $8.7 \pm 0.92$ skills correctly.

\section{DISCUSSION}

Opportunities for real-time training and evaluation of psychosocial intervention and referral skills may not present themselves during an athletic training student's clinical education, but these skills are essential for athletic trainers in clinical practice. Decision making and skill integration are best evaluated in a manner very similar to real-life clinical practice. ${ }^{9}$ The purpose of our study was to determine if a small-group SP encounter improved athletic training students' interpersonal communication, psychosocial intervention, and 
Table. Psychological Intervention and Referral

\begin{tabular}{lc}
\hline & $P$ Value \\
Skills & (Difference \\
Between \\
Ges No & Groups) \\
\hline
\end{tabular}

Interpersonal

Greeted patient warmly, confidently introduced self

Control

Experimental

190

182

.487

Used words I could understand (no medical jargon)

Control

Experimental

190

200

Called patient by name

Control

Experimental

145

$5 \quad 15$

Maintained eye contact much of the time but not excessively

Control

Experimental

172

182

.999

Used appropriate body language/ posture, sat on patient's level (eg, "nodding, leaning forward, facial expression")

Control

Experimental

$17 \quad 2$

200

.231

Listened with interest and did not interrupt while patient was talking

Control

Experimental

190

191

.999

Summarized information and verified my understanding

Control

Experimental

163

182

.661

Explained what he or she was about to do during the encounter (eg, "Now l'm going to examine ...")

Control

145

Experimental

173

.451

Was respectful, friendly, never judgmental, rude, arrogant, etc

Control

Experimental

181

191

NA

Respected modesty, personal

space, confidentiality

Control

Experimental

190

200

NA

Data-gathering - the examinee asked

questions about:

Perception of her weight

Control

Experimental
Table. Continued

$P$ Value

(Difference

Between

Skills

Yes No Groups)

Weight control methods

Control

Experimental

$\begin{array}{rr}5 & 14 \\ 14 & 6\end{array}$

.010

Coping strategies for stress

Control

Experimental

316

155

$.001^{*}$

Problem and treatment of muscle

cramps

Control

Experimental

$18 \quad 1$

191

Eating habits

Control

Experimental

154

164

.999

Frequency of vomiting (if

appropriate)

Control

Experimental

$0 \quad 19$

119

.999

Menstrual periods

Control

$0 \quad 19$

Experimental

$\begin{array}{ll}3 & 17\end{array}$

.231

Checked oral cavity

Control

$\begin{array}{ll}0 & 19\end{array}$

218

.487

Experimental

Made referral to counseling center, nutritionist, and/or physician

immediately

Control

Experimental

$5 \quad 14$

.105

119

Abbreviation: NA, not applicable.

* Indicates significant between-groups differences based on the Fisher exact text ( $\alpha=.003$ after Bonferroni adjustment).

referral skills. We found a small-group SP encounter significantly increased the psychosocial intervention and referral skills of athletic training students. To our knowledge no research has been published at this time examining the efficacy of educational interventions such as role play, simulation, or SP encounter(s) to improve the interpersonal communication and/or psychosocial intervention and referral skills of athletic training students. Specifically, our research demonstrates the educational value of a small-group SP encounter designed to improve psychosocial intervention and referral skills. During this encounter, the students actively engaged with the SP while receiving immediate guidance and feedback regarding their performance. Athletic training educators should consider using small-group SP encounters to teach psychosocial intervention and referral skills.

As previously mentioned, we chose a small-group SP encounter not only for the educational value but also because we feel this provides a rich method of teaching and might be the most feasible method by which faculty could implement 
SPs into athletic training education. Less faculty time and resources are needed to carry out small-group SP encounters versus individual SP encounters. Also, individual encounters are often used for evaluation rather than teaching and last anywhere from 15 to 30 minutes (scheduled in 30-minute intervals), and proper space (eg, small athletic training clinic, athletic training lab or office) is needed.

Small-group SP encounters have been used to teach interviewing and communication skills, clinical reasoning, and physical examination skills. ${ }^{18}$ Moreover, small-group SP encounters have been used to teach second-year medical students how to distinguish depression in older adults versus younger adults. ${ }^{21}$ Athletic training students in groups took turns interacting with an SP during different parts of the evaluation (eg, diagnosis, treatment plan, summary of visit). Once the information was gathered, the students worked together and discussed physical examination information and diagnostic tests, generated a diagnosis, and formulated a treatment plan. In a recent study ${ }^{20}$ where athletic training students evaluated an SP in groups, it was also found the students discussed their evaluation and treatment plan, thus engaging in peer-assisted learning during the small-group SP encounters.

Peer-assisted learning is the act of or process of gaining knowledge, understanding, or skill in athletic training tasks among students who are at either different or the same academic or experience levels through instruction and experience. ${ }^{22}$ During the small-group SP encounter, students discussed their evaluation process with others, and this exposed them to different approaches to their evaluation and counseling of patients. Although peer-assisted learning does occur naturally during clinical education, ${ }^{22}$ our participants engaged in intentional team/group activities. In the current competencies ${ }^{15}$ a team approach to practice is one of the foundational behaviors of professional practice. This small-group SP encounter provided an opportunity for the students to communicate with their peers regarding patient care. Part of a team approach to treatment and management of patients is communication. ${ }^{23}$ Communication is important with other health care providers as well as the patient.

Standardized patients have been shown to improve communication skills, ${ }^{24-26}$ but, interestingly, we did not find any significant improvement in interpersonal communication skills after the small-group SP encounter. The checklist items we used to evaluate the student were very general (eg, introduced self, made eye contact). Our senior athletic training students had interacted with many patients during their clinical experiences and performed various evaluations and treatments that likely provided them with the opportunity to develop these basic communication skills. We feel that if the interpersonal communication skills had been more sensitive and specific to psychosocial intervention and referral interpersonal communication (eg, did not feel judged, did not give false hope, was direct with potential options), significant improvements might have been found, as these communication skills are more specific to psychosocial intervention and referral. As mentioned, none of the participants had completed their psychosocial intervention and referral clinical proficiency evaluation before initiation of the study. Even if a student had done so during the semester, this still provides minimal experience and development of such communication skills.
This study uniquely contributes to the literature regarding the use of small-group SP encounters. We found improvements in certain psychosocial intervention and referral skills with 1 small SP group encounter. We did not study whether an individual encounter would improve psychosocial intervention and referral skills as well. The effect on learning and clinical performance of students interacting with the SP during the entire encounter versus only interacting with the SP during part of the encounter is also unknown. We used the time-in/time-out approach, ${ }^{18}$ where students together in their group interact intermittently with an SP. This method is similar to the start-stop method. With the start-stop method, students remain in groups ${ }^{17}$ but only 1 student interacts with the SP during different parts of the encounter (eg, history, physical examination, formulation of treatment plan) while the other students observe. Just as in the time-in/time-out approach, the teacher or student can stop the encounter when needed. We recommend comparing these different teaching interventions to determine which ones have good outcomes and are an efficient and effective use of time.

\section{Implications and Suggestions for Future Research}

Our study had some limitations, as it was performed in 1 athletic training program and the results may not be generalizable (eg, to other psychosocial intervention and referral skills for other conditions). It is possible those who completed the small-group SP encounter performed better on the individual SP encounter because of the additional experience in the small-group SP encounter. We did not track, nor were we able to control, when and if the athletic training students were exposed to any psychosocial intervention and referral encounters during their clinical experiences. The primary investigator, along with a clinician, using information from the literature and actual patient encounters in clinical practice, developed the cases. To ensure the case was evidence based, we also consulted the literature. We did not reference the literature we read and used when creating the case because we didn't build that into the case template. We recommend adding to the case template and referencing any literature that was used to help create SP cases. Future research should explore what volume of small-group SP encounters $(2-4+)$ for psychosocial intervention and referral can adequately prepare students to treat patients with a variety of psychological needs such as depression, addiction, and anxiety.

Future research should involve more participants, ideally from multiple athletic training programs. We used a checklist (yes, no) to evaluate performance. In future studies, a more global scale could be used with Likert scale items specific to each skill, such as needs improvement, adequately performed, skillfully performed or not asked, asked, asked in detail, which may be more sensitive to the performance of the student than yes or no responses. In the future, at the conclusion of the small-group SP encounters, focus groups could be used to understand the participants' experience during the encounter. Measuring the participants' anxiety levels before and after the small-group SP encounter could provide useful information as well.

We also recommend following up with the experimental group participants as to whether they perceived that the small-group SP encounter experience adequately prepared them for live patient encounters. Lastly, we recommend studies that explore 
the effects of small-group SP encounters in other areas of athletic training education that have limited opportunity for realistic or real-time practice, such as nutritional aspects of injury and illness, pharmacology, and/or general medical conditions and disabilities. ${ }^{9}$

\section{CONCLUSIONS}

Realistic experiences are needed in athletic training education, especially in the area of psychosocial intervention and referral, where students experience few to no live interactions with patients needing intervention and referral. As recently reported, SPs are used on a large scale in the training of health care professionals as well as during national licensing examinations and qualifying examinations. ${ }^{27}$ Our study was to determine if a small-group SP encounter improved athletic training students' interpersonal communication, psychosocial intervention, and referral skills. We found 1 small-group SP encounter improved the participants' psychosocial intervention and referral skills, but not their interpersonal communication. It would appear that small-group SP encounters could feasibly provide learning experiences of psychosocial intervention and referral of patients with signs of depression, eating disorder, and/or substance abuse that are realistic enough to be meaningful. Including SP encounters in psychosocial educational units of instruction may more adequately prepare students for treating real patients than educational programs that do not use SPs.

\section{REFERENCES}

1. Whitson EJ, Cordova ML, Demchak TJ, Stemmans CL, King KA. Certified athletic trainers' knowledge and perception of professional preparation involving eating disorders among athletes. J Allied Health. 2006;35(1):18-29.

2. Kessler RC, Wang PS. The descriptive epidemiology of commonly occurring mental disorders in the United States. Annu Rev Public Health. 2008;29:115-129.

3. Green G, Uryasz F, Petr T, Bray C. NCAA study of substance use and abuse habits of college student-athletes. Clin J Sport Med. 2001;11(1):51-56.

4. Yang J, Peek-Asa C, Corlette J, Cheng G, Foster D, Albright J. Prevalence of and risk factors associated with symptoms of depression in competitive collegiate student athletes. Clin J Sport Med. 2007;17(6):481-487.

5. Brenner J, Swanik K. High-risk drinking characteristics in collegiate athletes. J Am Coll Health. 2007;56(3):267-272.

6. Miller B, Miller M, Verhegge R, Linville H, Pumariega A. Alcohol misuse among college athletes: self-medication for psychiatric symptoms? J Drug Educ. 2002;32(1):41-52.

7. Bonci C, Bonci L, Granger L, et al. National Athletic Trainers' Association position statement: preventing, detecting, and managing disordered eating in athletes. J Athl Train. 2008; 43(1):80-108.

8. Armstrong KJ, Weidner TG, Walker SE. Athletic training approved clinical instructors report that more real-time opportunities are needed for evaluating clinical proficiencies. $J$ Athl Train. 2009;44(6):630-638.

9. Walker SE, Weidner TG, Armstrong KJ. Evaluation of athletic training students' clinical proficiencies. J Athl Train. 2008;43(4): 386-395.
10. Stiller-Ostrowski JL, Ostrowski J. Recently certified athletic trainers' undergraduate educational preparation in psychosocial intervention and referral. J Athl Train. 2009;44(1):6775 .

11. Weidner T, Henning J. Historical perspective of athletic training clinical education. J Athl Train. 2002;37(4 suppl):S222-S228.

12. Hamson-Utley JJ, Stiller-Ostrowski JL. Athletic training educators' instructional methods and confidence in graduating students' abilities regarding psychosocial intervention and referral. Athl Train Educ J. 2011;6(3):154-162.

13. Boulet J, de Champlain A, McKinley D. Setting defensible performance standards on OSCEs and standardized patient examinations. Med Teach. 2003;25(3):245-249.

14. Adamo G. Simulated and standardized patients in OSCEs: achievements and challenges 1992-2003. Med Teach. 2003;25(3): 262-270.

15. National Athletic Trainers' Association. Athletic Training Educational Competencies. 5th ed. Dallas, TX: National Athletic Trainers' Association; 2011.

16. Walker SE, Weidner TG. The use of standardized patients in athletic training education. Athl Train Educ J. 2010;5(2):87-89.

17. Howley LD1, Gliva-McConvey G, Thornton J. Standardized patient practices: initial report on the survey of US and Canadian medical schools. Med Educ Online. 2009;29(14):1-7.

18. Rethans JJ, Grosfeld FJ, Aper L, et al. Six formats in simulated and standardized patients use, based on experiences of 13 undergraduate medical curricula in Belgium and the Netherlands. Med Teach. 2012;34(9):710-716.

19. Barrows H. An overview of the uses of standardized patients for teaching and evaluating clinical skills. Acad Med. 1993;68(6): 443-445.

20. Walker SE, Weidner TG, Armstrong KJ. Standardized patient encounters and individual case-based simulations improve students' confidence and promote reflection: a preliminary study. Athl Train Educ J. 2015;10(2):130-137.

21. Farrell TW. Review of "depression in the elderly-simulated patient small group activity." J Am Geriatr Soc. 2012;60(1):142144.

22. Henning JM, Weidner TG, Jones J. Peer-assisted learning in the athletic training clinical setting. J Athl Train. 2006;41(1):102-108.

23. Interprofessional Education Collaborative Expert Panel. Core Competencies for Interprofessional Collaborative Practice: Report of an Expert Panel. Washington, DC: Interprofessional Education Collaborative; 2011.

24. Lin EC, Chen SL, Chao SY, Chen YC. Using standardized patient with immediate feedback and group discussion to teach interpersonal and communication skills to advanced practice nursing students. Nurse Educ Today. 2013;33(6):677683.

25. Mesquita AR, Lyra DP Jr, Brito GC, Balisa-Rocha BJ, Aguiar PM, de Almeida Neto AC. Developing communication skills in pharmacy: a systematic review of the use of simulated patient methods. Patient Educ Couns. 2010;78(2):143-148.

26. Schlegel C, Woermann U, Shaha M, Rethans JJ, van der Vleuten C. Effects of communication training on real practice performance: a role-play module versus a standardized patient module. J Nurs Educ. 2012;51(1):16-22.

27. Swanson DB, van der Vleuten CPM. Assessment of clinical skills with standardized patients: state of the art revised. Teach Learn Med. 2013;25(suppl 1):S17-S25. 